\title{
Long-read DNA sequencing fully characterized chromothripsis in a patient with Langer-Giedion syndrome and Cornelia de Lange syndrome-4
}

\author{
Ming Lei ${ }^{1,2,3} \cdot$ Desheng Liang ${ }^{1} \cdot{\text { Yifeng } \text { Yang }^{4} \cdot \text { Satomi Mitsuhashi }}^{2} \cdot$ Kazutaka Katoh $^{5,6} \cdot$ Noriko Miyake $^{2}$. \\ Martin C. Frith ${ }^{6,7,8} \cdot$ Lingqian Wu $(1)^{1} \cdot$ Naomichi Matsumoto $^{2}$
}

Received: 18 February 2020 / Revised: 18 March 2020 / Accepted: 18 March 2020 / Published online: 15 April 2020

(c) The Author(s) 2020. This article is published with open access

\begin{abstract}
Chromothripsis is a type of chaotic complex genomic rearrangement caused by a single event of chromosomal shattering and repair processes. Chromothripsis is known to cause rare congenital diseases when it occurs in germline cells, however, current genome analysis technologies have difficulty in detecting and deciphering chromothripsis. It is possible that this type of complex rearrangement may be overlooked in rare-disease patients whose genetic diagnosis is unsolved. We applied long read nanopore sequencing and our recently developed analysis pipeline dnarrange to a patient who has a reciprocal chromosomal translocation $\mathrm{t}(8 ; 18)(\mathrm{q} 22 ; \mathrm{q} 21)$ as a result of chromothripsis between the two chromosomes, and fully characterize the complex rearrangements at the translocation site. The patient genome was evidently shattered into 19 fragments, and rejoined into derivative chromosomes in a random order and orientation. The reconstructed patient genome indicates loss of five genomic regions, which all overlap with microarray-detected copy number losses. We found that two disease-related genes RAD21 and EXT1 were lost by chromothripsis. These two genes could fully explain the disease phenotype with facial dysmorphisms and bone abnormality, which is likely a contiguous gene syndrome, Cornelia de Lange syndrome type IV (CdLs-4) and atypical Langer-Giedion syndrome (LGS), also known as trichorhinophalangeal syndrome type II (TRPSII). This provides evidence that our approach based on long read sequencing can fully characterize chromothripsis in a patient's genome, which is important for understanding the phenotype of disease caused by complex genomic rearrangement.
\end{abstract}

These authors contributed equally: Ming Lei, Desheng Liang, Yifeng Yang

Supplementary information The online version of this article (https:// doi.org/10.1038/s10038-020-0754-6) contains supplementary material, which is available to authorized users.

\footnotetext{
Lingqian $\mathrm{Wu}$

wulingqian@sklmg.edu.cn

$\triangle$ Naomichi Matsumoto naomat@yokohama-cu.ac.jp

1 Center for Medical Genetics \& Hunan Key Laboratory of Medical Genetics, School of Life Sciences, Central South University, Changsha, Hunan, China

2 Department of Human Genetics, Yokohama City University Graduate School of Medicine, Yokohama, Japan

3 China Astronaut Research and Training Center, Beijing, China
}

\section{Introduction}

Chromothripsis, a chaotic complex genomic rearrangement, may cause tumorigenesis and congenital disorders if it occurs somatically and constitutionally, respectively. Chromothripsis arises in the genome as a single event and causes many shattered fragments of the genome which

4 Department of Cardiovascular Surgery, The Second Xiangya Hospital of Central South University, Changsha, China

5 Research Institute for Microbial Diseases, Osaka University, Suita, Japan

6 Artificial Intelligence Research Center, National Institute of Advanced Industrial Science and Technology (AIST), Tokyo, Japan

7 Graduate School of Frontier Sciences, University of Tokyo, Chiba, Japan

8 Computational Bio Big-Data Open Innovation Laboratory (CBBD-OIL), AIST, Tokyo, Japan 
rejoin into rearranged derivative chromosome(s) [1]. Structures of derivative chromosomes from chromothripsis may become very complex. It is usually hard to understand how these fragments are ordered in a patient's genome. There are approaches to understand the whole structure of chromothripsis using DNA sequencers, however, it is still challenging to completely understand structural variations from short DNA reads [2]. New approaches use long read sequencers (i.e., Oxford Nanopore Technologies' nanopore sequencers, hereafter nanopore, or PacBio sequencers) [2], because, in principle, longer reads have advantages in detecting structural variations, since they have better coverage of repetitive regions, which occupy nearly half of the human genome and can be the source of rearrangements (e.g., Alu/Alu mediated recombination [3]). In addition, long reads may contain multiple breakpoints in one read, which helps to find the order and orientation of the fragments [4]. However, even if all breakpoints are detected, painstaking manual inspection is still needed to reconstruct whole rearrangements. We recently described a method for reconstructing complex rearrangements from long read sequencing data by an automatic algorithm, which may infer the full structure of the patient's genomic rearrangement [4]. Using this approach, we could fully characterize derivative chromosomes created by chromothripsis in a patient with a congenital disease.

\section{Materials and methods}

\section{Nanopore sequencing using PromethION}

DNA was extracted from the patient's blood cells. Library was prepared for nanopore sequencing using DNA ligation kit (SQK-LSK109) then subjected to PromethION sequencing (Oxford Nanopore Technologies) using one PRO-002 (R9.4.1) flowcell according the manufacturer's protocol. Base-calling and fastq conversion were performed with MinKNOW ver1.14.2. Control datasets were also sequenced PromethION as previously described [4]. Base-calling and fastq conversion were performed with MinKNOW ver1.11.5.

\section{dnarrange}

Long nanopore reads were aligned to the human reference genome (GRCh38) using LAST as described here (https:// github.com/mcfrith/last-rna/blob/master/last-long-reads.

$\mathrm{md})$. The analysis pipeline for finding and characterizing rearrangements is described elsewhere [4]. Briefly, rearrangements were detected using dnarrange (https://github. $\mathrm{com} / \mathrm{mcfrith} /$ dnarrange) and we filtered patient-only rearrangements using 33 control datasets [4]. Next, we merged each group of overlapping rearranged reads into a consensus sequence, using lamassemble (https://gitlab.com/ mcfrith/lamassemble), and realigned to the reference genome. We used dnarrange-link, an algorithm to infer the order and orientation of multiple rearrangements, to understand the whole chromothripsis.

\section{Sanger-sequence confirmation of breakpoints}

PCR primers for breakpoints were designed using primer3 software (http://bioinfo.ut.ee/primer3-0.4.0/). Primers used are shown in Table S3. PCR amplification was done using ExTaq (Takara), then amplified products were Sanger sequenced using BioDye Terminater v3.1 Cycle Sequencing kit with 3130xl genetic analyzer (Applied Biosystems, CA, USA). Sanger electropherograms were visualized using Sequencher (Gene Codes Corporation, MI, USA).

\section{Gene expression levels in lymphoblastoid cell}

Total RNA was extracted from lymphoblastoid cells from the patient and controls using RNeasy Plus Mini Kit (QIAGEN, Hilden, Germany), then subjected to reversetranscription reaction using SuperScriptIII (Thermo Fisher Scientific). Quantitative real-time PCR was performed using Rotor-Gene SYBR Green PCR Kit and Rotor-Gene Q (IAGEN, Hilden, Germany). Delta Delta CT method was used to compare gene expression levels. Primers used are shown in Table S5.

\section{Results}

The patient is a 6-year-old boy with facial dysmorphism, congenital heart defect, thoracic scoliosis, clinodactyly of fifth fingers, fingerpads, and intellectual disability (ID). He was the first child of healthy, unrelated parents of HanChinese origin with unremarkable family history, with a karyotype of 46,XY,t(8;18)(q22;q21)dn (Fig. 1a). He was born at term via normal spontaneous vaginal delivery. After birth, he was noted to show growth retardation, facial dysmorphism, cleft palate (left side), and congenital heart defects. He was referred to our clinical genetics laboratory at 6 years of age. Physical examination showed: height 101 $\mathrm{cm}(-2.0 \mathrm{SD})$, weight $12.5 \mathrm{~kg}(-2.0 \mathrm{SD})$. He had mild-tomoderate ID, congenital heart defect (ventricular septal defect, right ventricle double exit, aorta, and pulmonary artery juxtaposition), thoracic scoliosis, butterfly vertebra (T5, T8, T10, and T12), clinodactyly of fifth fingers, fingerpads, umbilical hernia (left side), inguinal hernia, recurrent respiratory infections, together with facial dysmorphism including arched, thick eyebrows, long eyelashes, depressed nasal bridge, anteverted nostrils, large prominent ears, dental malocclusion, and micrognathia. At 
A $46, \mathrm{XY}, \mathrm{t}(8 ; 18)(\mathrm{q} 22 ; \mathrm{q} 21)$

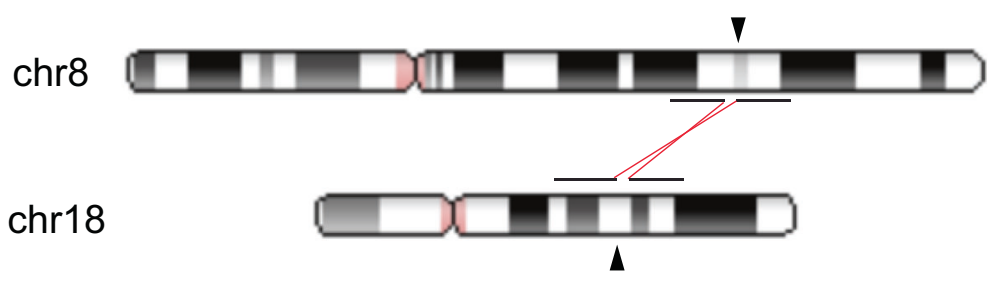

B

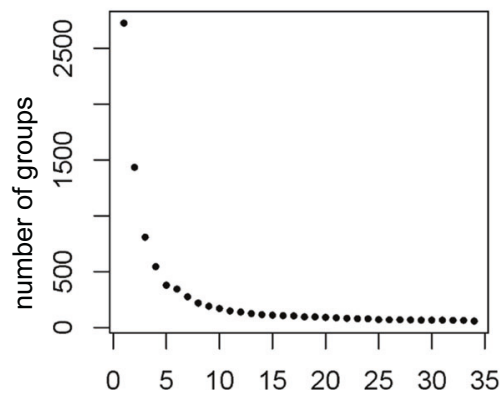

C

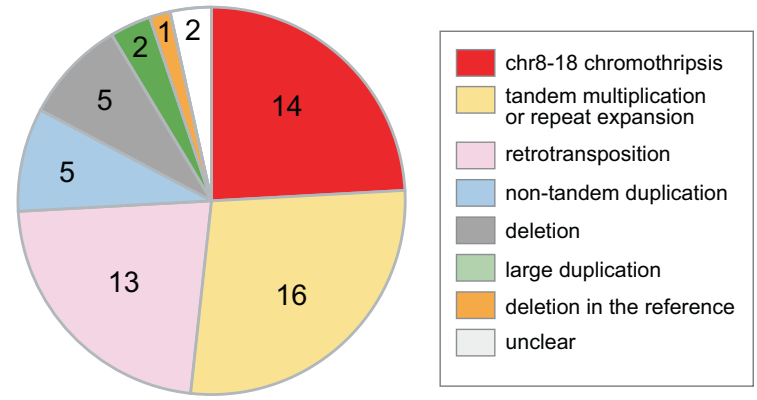

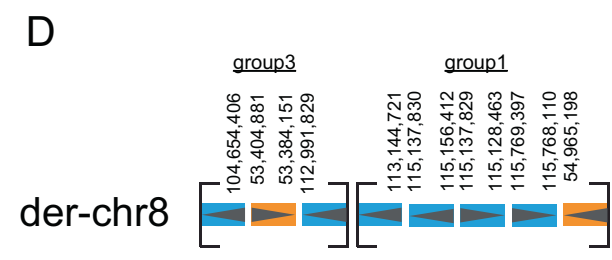

2726 groups

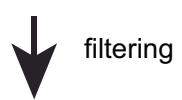

59 groups

chr8, chr18

14 groups
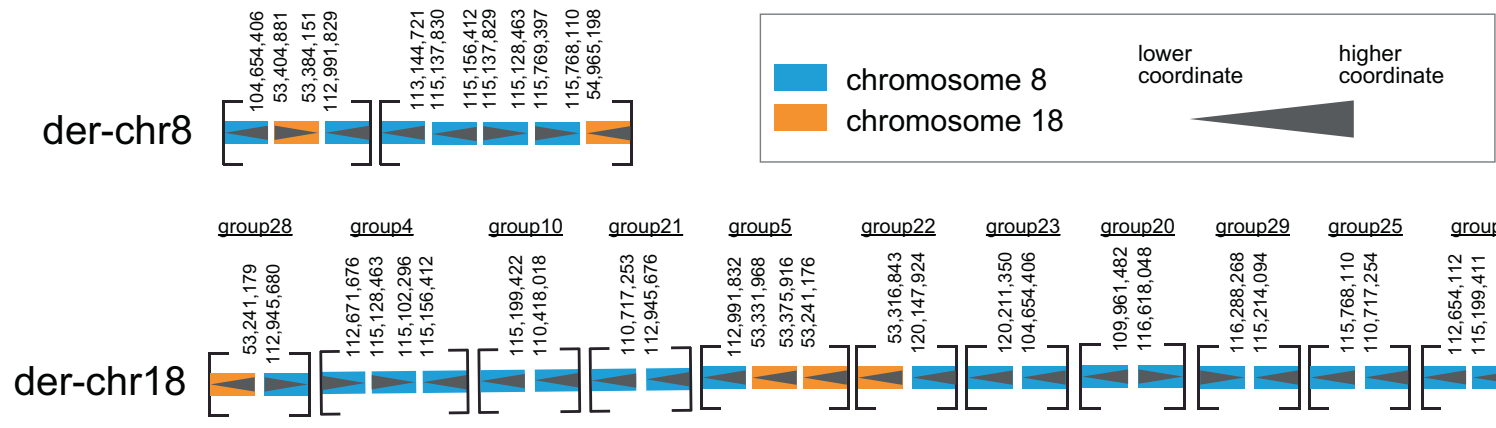

Fig. 1 Detection strategy of the patient's complex rearrangement. a Ideograms of translocation position of the patient. b Filtering out rearrangements shared with 33 controls finds 59 groups of reads with patient-only rearrangements. The plot shows exponential decrease of the number of groups, by successive subtraction of shared rearrangements using 33 control datasets. $y=$ the number of groups, $x=$

the time, he was suspected to have Kabuki syndrome. He was found to have multiple exostoses in his right leg, left calf and shoulder blade at the age of 10 years. Further details of the later phenotype including a picture of the face at the age of 10 were described previously [5].

We performed short read whole-genome sequencing with 150-bp paired-end reads (Illumina) using the genomic DNA from lymphoblastoid cell line (LCL) of the patient. Reads were aligned to the human genome reference (hg19), and candidate breakpoints were predicted using Breakdancer1.45 [6] as previously described [7]. However, there were too many breakpoint candidates and we could not figure out the chr8-18 reciprocal chromosomal translocation (Table S1), compared with our previous study dealing with simple reciprocal chromosomal translocations in which BreakDancer could detect only one candidate breakpoint at the translocation site [7]. We suspected this patient might have very complex chromosomal rearrangements, such as chromothripsis. We did not find any pathogenic variants (single nucleotide change or a few nucleotides deletions/ insertions) in Kabuki syndrome related genes (KMT2D; OMIM\#147920, KDM6A; OMIM\#300867).

Next we sequenced genomic DNA from LCLs of the patient using a nanopore long read sequencer, PromethION, 
and obtained 10,397,629 long reads with $119 \mathrm{G}$ bases (predicted $\times 36$ coverage) of mean length of 11,451 bases and median length of 6780 bases. We recently developed a new analytic pipeline, dnarrange, to detect rearrangements from long read sequencing data, and order and orient multiple DNA fragments to reconstruct complex changes in derivative chromosomes [4]. We applied dnarrange to find groups of long reads that overlap the same rearrangement, and then filtered out "normal or nonpathogenic" rearrangements that are shared by any of 33 individuals without the same disease, as described previously [4] (Fig. 1b). The number of groups of rearranged reads decreased to 59 by subtracting rearrangements shared with controls (Fig. 1b). The initial exponential decrease (Fig. 1b) suggests that many rearrangements are shared with other individuals, or are reference-specific rearrangements. We characterized all patient-specific rearrangements (Table S2, Fig. S1). A large fraction of them are tandem multiplications $(N=6) /$ tandem repeat expansions $(N=10)(N=16 / 59,27 \%)$ or retrotransposon insertions (L1HS $(N=11)$, SVA $(N=1)$ and AluYb8 $(N=1))(N=13 / 59,22 \%)$ (Figs. 1c, S1, and Table S2). These types of retrotransposon are recently integrated into human genomes and they are still active or polymorphic in the population, thus they were not filtered out using 33 controls [8]. A striking finding is that 14 groups of rearranged reads, the second largest fraction $(N=$ $14 / 59,24 \%)$, are involved in the patient's chromosomal translocation $\mathrm{t}(8 ; 18)(\mathrm{q} 22 ; \mathrm{q} 21)$ (Figs. 1c, S2). We merged the reads in each group into consensus sequences, using lamassemble [4] (Fig. S2), and estimated rearrangement breakpoints as previously described [4]. We also confirmed all the breakpoints by Sanger sequencing (Fig. S3, Table S3). Next we applied dnarrange-link, and found a unique way to order these 14 groups (Fig. 1d) [4]. The two reconstructed derived chromosomes contain 19 rearranged fragments (Fig. 2a, numbered): 15 fragments are from chr8
Fig. 2 Reconstruction of patient's chromothripsis. a Patient's derivative chromosome 8 (der[8]) and 18 (der[18]) are compared with the reference genome, hg38. Horizontal dotted gray lines join the parts of each derivative chromosome. Insets enlarge tiny fragments in der-chr8 and der-chr18. Numbers label fragments (breakpoint-to-breakpoint). From this picture, 15 fragments are from chr8 [1-5,8-13,16-19] and 4 fragments are from chr18 $[6,7,14,15]$. Derivative chromosome 8 has 14 fragments and 18 has 5 fragments. b Vertical dotted gray lines join fragments that come from adjacent parts of the reference genome. Yellow rectangles show deletions. There are four deletions in chr8 and one deletion in chr18
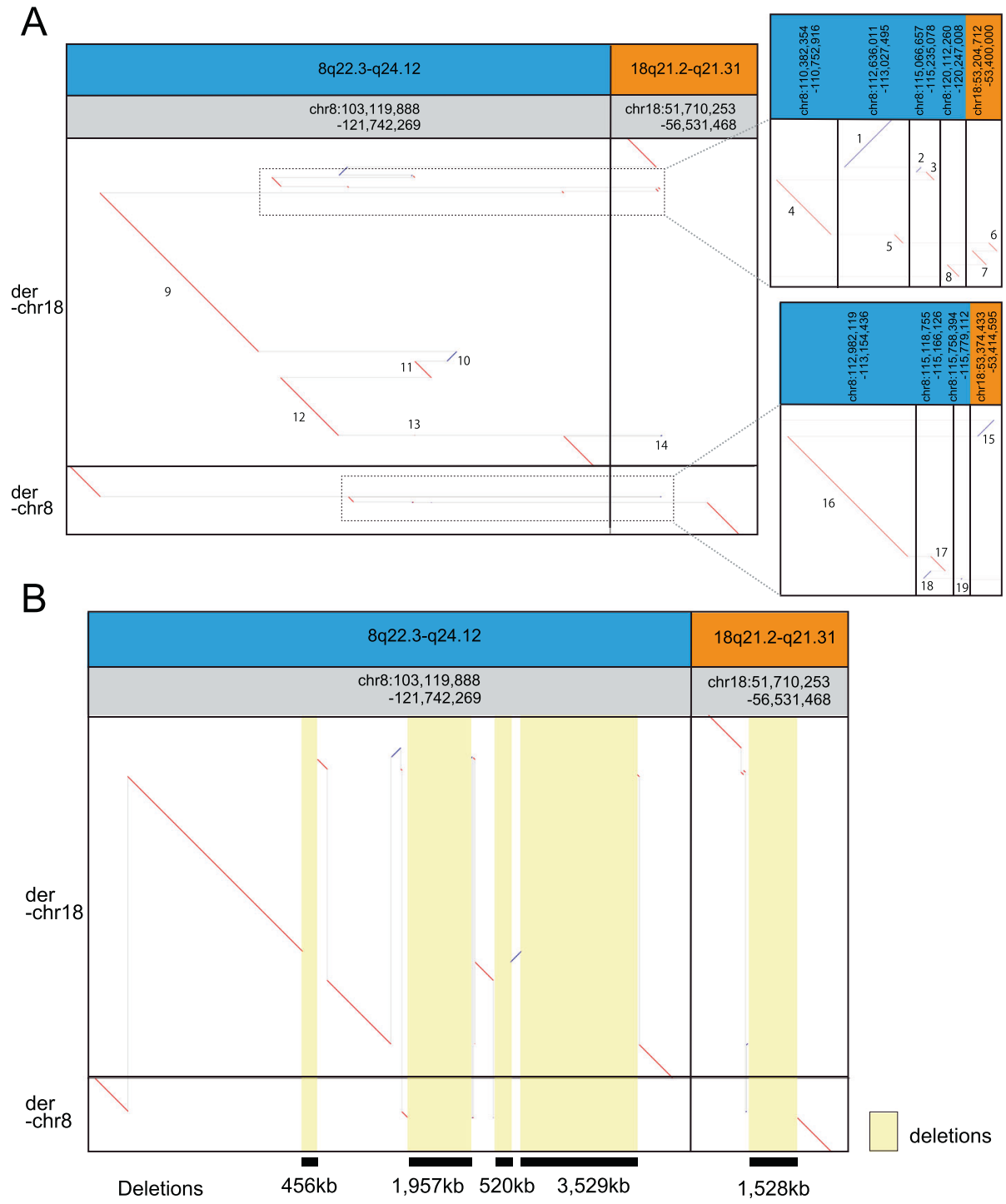
and 4 fragments are from chr18. Derivative chromosome 8 contains 14 of these fragments, and derivative chromosome 18 contains 5. The rearrangement involves four large deletions from chr8 $(456,1,957,520$, and $3529 \mathrm{~kb})$ and one large deletion from chr18 (1528 kb) (Fig. 2b, yellow rectangles). We compared these five deletions with CGH array results [5]. The five deletion loci all agreed in both methods, but SNP array data showed smaller deletion sizes than the reconstructed sequence due to its low probe resolution (Table S4). These deletions can only be inferred from the fully reconstructed rearrangement, and not from any part of the rearrangement [4]. This is an important property of reconstructing complex rearranged sequences by our method.

We noted that one deletion from chr8 contained disease associated genes, RAD21 and EXT1 (Figs. 3a, S4). Realtime PCR results from the LCLs of the patient showed decreased gene expression of RAD21 (OMIM 606462) and EXT1 (OMIM 608177) but not TRPS1 (OMIM 604386) (Fig. 3b, Table S5).

\section{Discussion}

We report a patient with chromothripsis at the $\mathrm{t}(8 ; 18)$ translocation site which led to the loss of RAD21 and EXT1. Disruptions of RAD21 and EXT1 genes are known to cause rare developmental diseases. Langer-Giedion syndrome (LGS), also known as Trichorhinophalangeal Syndrome Type II (TRPSII) (OMIM 150230), is a rare contiguous gene syndrome caused by a deletion involving loss of functional copies of TRPS1 and EXT1 [9, 10]. LGS/TRPSII is characterized by short stature, microcephaly, sparse scalp hair, bulbous nose, long flat philtrum, thin upper vermilion,

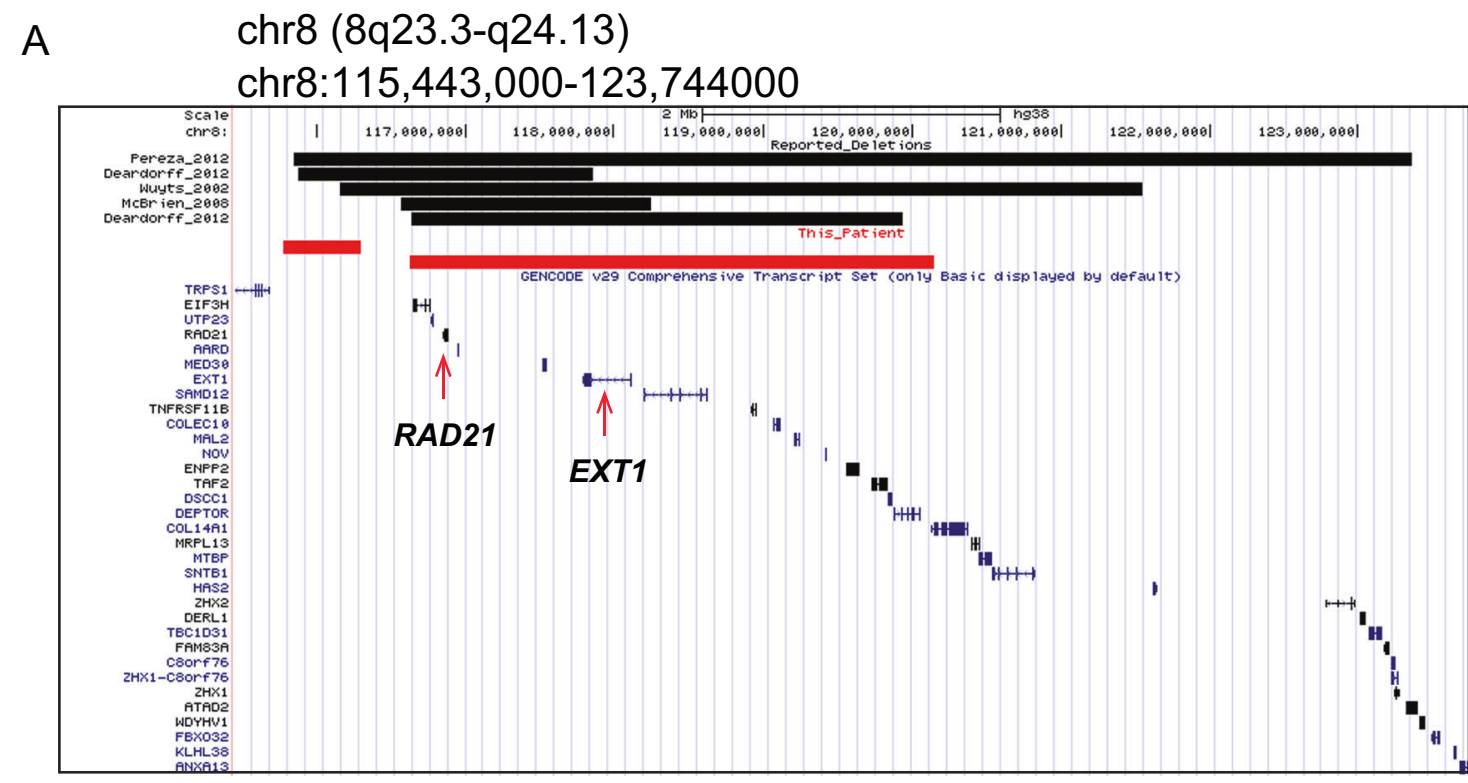

B

TRPS1

EXT1

RAD21
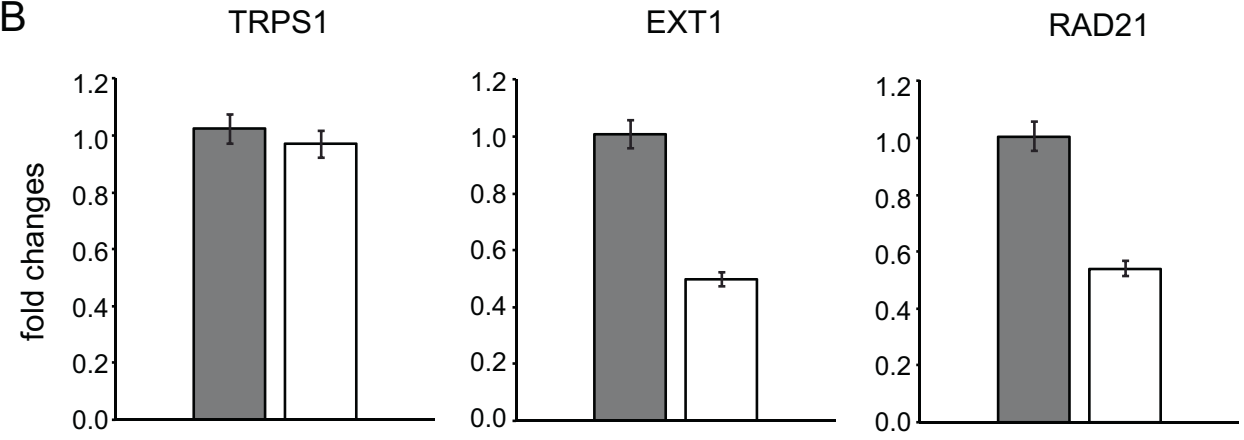

Fig. 3 Deletion of RAD21-EXT1 but not TRPS1. a Five reported patients have deletions (black bars) disrupting RAD21 and EXT1. This patient also has deletions (red bars) in the same region. b Quantitative RT-PCR shows decreased expression of RAD21 and EXT1. The

expression level of TRPS1 is not altered. Error bars: standard deviations from three experiments. Controls are three different individuals without the same disease 
Table 1 The clinical features of our patient compared to CdLs4, LGS/TRPS II and Kabuki Syndrome

\begin{tabular}{|c|c|c|c|c|c|}
\hline & Patient & $\begin{array}{l}\text { CdLS-4 with RAD21 } \\
\text { pathogenic variants }\end{array}$ & CdLS & $\begin{array}{l}\text { LGS/ } \\
\text { TRPSII }\end{array}$ & $\begin{array}{l}\text { Kabuki } \\
\text { syndrome }\end{array}$ \\
\hline \multicolumn{6}{|l|}{ Facial dysmorphism } \\
\hline Arched, thick eyebrows & + & + & + & + & + \\
\hline Long eyelashes & + & + & + & - & + \\
\hline Depressed nasal bridge & + & + & + & + & + \\
\hline Anteverted nostrils & + & + & + & - & - \\
\hline Large prominent ears & + & - & - & + & + \\
\hline Cleft palate & + & + & ${ }^{+}(20 \%)$ & ${ }^{+}$(Rare) & ${ }^{+}($Rare $)$ \\
\hline Dental malocclusion & + & ${ }^{+}($Rare $)$ & + & ${ }^{+}($Rare $)$ & + \\
\hline Micrognathia/mandibular hypoplasia & + & ${ }^{+}($Rare $)$ & + & + & - \\
\hline \multicolumn{6}{|l|}{ Skeletal } \\
\hline Multiple exostosis & + & - & - & + & - \\
\hline Small hands and feet & + & ${ }^{+}($Rare $)$ & + & - & - \\
\hline Finger pads & + & ${ }^{+}($Rare $)$ & - & ${ }^{+}$(Rare) & + \\
\hline Clinodactyly of the fifth fingers & + & + & + & ${ }^{+}($Rare $)$ & - \\
\hline Scoliosis: thoracic & + & - & ${ }^{+}(1 / 3)$ & + & + \\
\hline \multicolumn{6}{|l|}{ Respiratory } \\
\hline Recurrent respiratory infections & + & ${ }^{+}$(Rare) & + & + & - \\
\hline \multicolumn{6}{|l|}{ Cardiovascular } \\
\hline Congenital heart defect & + & ${ }^{+}$(Rare) & ${ }^{+}(25 \%)$ & - & + \\
\hline \multicolumn{6}{|l|}{ Neurologic } \\
\hline Intellectual disability & + & + & + & + & + \\
\hline
\end{tabular}

large prominent ears, multiple exostoses, cone-shaped epiphyses of phalanges and mild-to-severe ID. Later it was reported that dominant pathogenic variants in $R A D 21$, which resides between TRPS1 and EXT1, cause Cornelia de Lange syndrome type IV (CdLs-4) (OMIM 614701) [11]. Thus they are considered to be overlapping diseases. Cornelia de Lange syndrome (CdLs) is a multisystem disorder characterized by multiple facial and limb anomalies and ID $[12,13]$. To date, there are seven genes known to cause CdLs (NIPBL, SMC1A, SMC3, RAD21, BRD4, HDAC8, and $A N K R D 11)$ [13]. Pathogenic variants in $R A D 21$ at 8q24.11 cause CdLs-4 (OMIM 614701) [11]. CdLs-4 is characterized by synophrys, long micrognathia, brachydactyly, short stature, vertebral anomalies, and ID [11, 13] (Table 1).

Interestingly, five patients with Langer-Giedion syndrome without TRPS1 gene disruption have been reported (Fig. 3a) [11, 14-16]. Consistent with our patient, all five patients have deletions involving RAD21 and EXT1. The six individuals including ours have overlapping phenotypes: facial dysmorphism commonly included arched thick eyebrows (six patients), long eyelashes (five patients), sparse and thin scalp hair (four patients), downslanted palpebral fissures (three patients), depressed/broad nasal bridge (five patients), large prominent ears (two patients), long and flat philtrum (three patients), thin upper lip vermilion (four patients), cleft palate (two patients), micrognathia (three patients), and microcephaly (four patients). Skeletal symptoms from the six patients included short stature (two patients), clinodactyly of the fifth fingers/toes (two patients), and fat pads in fingers/toes (two patients). Four of six individuals had ID, and two of six had recurrent respiratory infections (Table 2 ). In addition to these symptoms, our patient had thoracic scoliosis and congenital heart defect, which were not described in the five patients previously reported. Previous study on this patient implied the possible involvement of ZFPM2 because of his cardiac phenotype [5], but we could not find disruption of this gene nor alteration of its transcript level, thus we concluded that involvement of ZFPM2 is unlikely (Fig. S5). Interestingly, among eight cases of $R A D 1$ pathogenic variants, two had congenital heart disease $[11,17]$. It is possible that $R A D 21$ defect might be responsible for this patient's congenital heart disease.

Several other genes were disrupted by his complex rearrangements (Fig. S4, Table S6), but none of them are related to this patient's phenotype based on OMIM. All symptoms were explained by LGS and CdLs-4 (Table 1), thus we speculate that disruption of other genes may have no or minimum effect on this patient's phenotype. (Fig. 3). The patient was first suspected of Kabuki syndrome because of a few overlapping phenotypes (Table 1). By genomic analysis, we could determine that this patient can be clearly categorized into LGS and CdLs-4. 
Table 2 The clinical features of our patient compared to 5 previously reported patients

\begin{tabular}{|c|c|c|c|c|c|c|}
\hline & This patient & $\begin{array}{l}\text { Wuyts et al. } \\
{[14]}\end{array}$ & $\begin{array}{l}\text { McBrien et al. } \\
{[15]}\end{array}$ & $\begin{array}{l}\text { Pereza et al. } \\
{[16]}\end{array}$ & $\begin{array}{l}\text { Deardorff et al. } \\
\text { [11] }\end{array}$ & $\begin{array}{l}\text { Deardorff et al. } \\
\text { [11] }\end{array}$ \\
\hline Deletion size & $\begin{array}{l}0.5 \mathrm{MB} \\
3.5 \mathrm{Mb}\end{array}$ & $5.4 \mathrm{Mb}$ & $1.69 \mathrm{Mb}$ & $7.5 \mathrm{Mb}$ & $3.3 \mathrm{MB}$ & $2.0 \mathrm{MB}$ \\
\hline TRPS1 & Not deleted & Not deleted & Not deleted & Not deleted & Not deleted & Not deleted \\
\hline$R A D$ & Deleted & Deleted & Deleted & Deleted & Deleted & Deleted \\
\hline EXT1 & Deleted & Deleted & Deleted & Deleted & Deleted & Deleted \\
\hline \multicolumn{7}{|l|}{ Facial dysmorphism } \\
\hline Sparse and thin scalp hair & - & + & + & + & + & NA \\
\hline Arched, thick eyebrows & + & + & + & + & + & + \\
\hline Long eyelashes & + & - & + & + & + & + \\
\hline Downslanted palpebral fissures & + & + & NA & + & NA & NA \\
\hline Bulbous tip of the nose & - & - & - & + & - & - \\
\hline Depressed/broad nasal bridge & + & + & - & + & + & + \\
\hline Large prominent ears & + & NA & - & + & NA & NA \\
\hline Long and flat philtrum & - & + & - & + & - & + \\
\hline Thin upper lip vermilion & - & + & + & + & - & + \\
\hline Cleft palate/high palate & + & - & - & - & + & - \\
\hline Micrognathia/mandibular hypoplasia & + & + & - & - & + & - \\
\hline Microcephaly & - & + & + & - & + & + \\
\hline \multicolumn{7}{|l|}{ Skeletal } \\
\hline Short stature & + & - & - & - & + & + \\
\hline Multiple exostoses & + & + & + & + & + & + \\
\hline Scoliosis & + & - & - & - & - & - \\
\hline Cone-shaped epiphyses & - & - & - & + & - & - \\
\hline Clinodactyly of the fifth fingers/toes & + & - & - & - & + & - \\
\hline Fat pads in fingers/toes & + & - & + & - & - & - \\
\hline \multicolumn{7}{|l|}{ Others } \\
\hline Recurrent respiratory infections & + & NA & + & NA & NA & NA \\
\hline Congenital heart defect & + & - & - & - & - & - \\
\hline Intellectual disability & + & + & + & + & - & - \\
\hline
\end{tabular}

In conclusion, we report a boy with phenotypes overlapping LGS and CdLs-4 caused by complex chromosomal rearrangements involving chr8 and chr18. We fully characterized the patient's germline chromothripsis using whole-genome nanopore long read sequencing and a new analysis pipeline dnarrange, thereby reconstructing the patient's chromosomal rearrangement from multiple DNA fragments. Our finding suggests that long read sequencing may be considered if conventional short read sequencing fails to detect breakpoints in patients who are suspected to have chromosomal rearrangements. It is of note that LGS is caused by large deletions, however, due to the limited number of patients and lack of complete characterization of deleted regions (i.e., usually they are characterized only partly by copy number analysis without precise breakpoint detection), genotype-phenotype correlations in this disease are not clear (i.e., which genes contribute to what extent of the phenotype). It is important to completely characterize each patient's genomic structure, and compare it to the gene-specific phenotypes caused by pathogenic variants in each gene.

\section{Web resources}

LAST: http://last.cbrc.jp, MAFFT: https://mafft.cbrc.jp, lamassemble: https://gitlab.com/mcfrith/lamassemble, dnarrange: https://github.com/mcfrith/dnarrange, NCBI genome decoration: https://www.ncbi.nlm.nih.gov/genome/tools/gdp, UCSC genome browser: https://genome.ucsc.edu/.

Acknowledgements This work was supported by the National Natural Science Foundation of China under the grant numbers 81771599 and 31601035 (to LW); AMED under the grant numbers JP19ek0109280, JP19dm0107090, JP19ek0109301, JP19ek0109348 and JP18kk020501 (to NMa); JSPS KAKENHI under the grant numbers JP17H01539 (to NMa), JP19K07977 (to SM) and JP19H03621 (to NMi); grants from the Ministry of Health, Labor, and Welfare under the grant 
numbers 30-6 and 30-7 (to NMa); and the Takeda Science Foundation (to NMi and NMa).

\section{Compliance with ethical standards}

Conflict of interest The authors declare that they have no conflict of interest.

Ethical approval The Ethics Committees of Hunan Jiahui Genetics Hospital and Yokohama City University approved the experimental protocols for genetic analysis. Written informed consent was obtained from the patient's parents for genetic investigation. All the methods were performed in accordance with the Declaration of Helsinki.

Publisher's note Springer Nature remains neutral with regard to jurisdictional claims in published maps and institutional affiliations.

Open Access This article is licensed under a Creative Commons Attribution 4.0 International License, which permits use, sharing, adaptation, distribution and reproduction in any medium or format, as long as you give appropriate credit to the original author(s) and the source, provide a link to the Creative Commons license, and indicate if changes were made. The images or other third party material in this article are included in the article's Creative Commons license, unless indicated otherwise in a credit line to the material. If material is not included in the article's Creative Commons license and your intended use is not permitted by statutory regulation or exceeds the permitted use, you will need to obtain permission directly from the copyright holder. To view a copy of this license, visit http://creativecommons. org/licenses/by/4.0/.

\section{References}

1. Kloosterman WP, Cuppen E. Chromothripsis in congenital disorders and cancer: similarities and differences. Curr Opin Cell Biol. 2013;25:341-8.

2. Sanchis-Juan A, Stephens J, French CE, Gleadall N, Megy K, Penkett C, et al. Complex structural variants in Mendelian disorders: identification and breakpoint resolution using short- and long-read genome sequencing. Genome Med 2018;10:95.

3. Zhu W, Mitsuhashi S, Yonekawa T, Noguchi S, Huei JC, Nalini A, et al. Missing genetic variations in GNE myopathy: rearrangement hotspots encompassing 5'UTR and founder allele. J Hum Genet. 2017;62:159-66.

4. Mitsuhashi S, Ohori S, Katoh K, Frith MC, Matsumoto N. A method for complete characterization of complex germline rearrangements from long DNA reads. medRxiv2019. https://doi. org/10.1101/19006379.

5. Tan ZP, Huang C, Xu ZB, Yang JF, Yang YF. Novel ZFPM2/ FOG2 variants in patients with double outlet right ventricle. Clin Genet. 2012;82:466-71.

6. Fan X, Abbott TE, Larson D, Chen K. BreakDancer: identification of genomic structural variation from paired-end read mapping. Curr Protoc Bioinforma. 2014;45:15.6.1-11.

7. Lei M, Mitsuhashi S, Miyake N, Ohta T, Liang D, Wu L, et al. Translocation breakpoint disrupting the host SNHG14 gene but not coding genes or snoRNAs in typical Prader-Willi syndrome. J Hum Genet. 2019;64:647-52.

8. Stewart C, Kural D, Stromberg MP, Walker JA, Konkel MK, Stutz AM, et al. A comprehensive map of mobile element insertion polymorphisms in humans. PLoS Genet 2011;7:e1002236.

9. Schinzel A, Riegel M, Baumer A, Superti-Furga A, Moreira LM, Santo LD, et al. Long-term follow-up of four patients with Langer-Giedion syndrome: clinical course and complications. Am J Med Genet A. 2013;161A:2216-25.

10. Vaccaro M, Tchernev G, Wollina U, Lotti T, Guarneri C. Trichorhinophalangeal syndrome. Open Access Maced J Med Sci. 2017;5:486-9.

11. Deardorff MA, Wilde JJ, Albrecht M, Dickinson E, Tennstedt S, Braunholz D, et al. RAD21 mutations cause a human cohesinopathy. Am J Hum Genet. 2012;90:1014-27.

12. Jackson L, Kline AD, Barr MA, Koch S. de Lange syndrome: a clinical review of 310 individuals. Am J Med Genet. 1993;47:940-6.

13. Kline AD, Moss JF, Selicorni A, Bisgaard AM, Deardorff MA, Gillett PM, et al. Diagnosis and management of Cornelia de Lange syndrome: first international consensus statement. Nat Rev Genet. 2018;19:649-66.

14. Wuyts W, Roland D, Ludecke HJ, Wauters J, Foulon M, Van Hul $\mathrm{W}$, et al. Multiple exostoses, mental retardation, hypertrichosis, and brain abnormalities in a boy with a de novo $8 \mathrm{q} 24$ submicroscopic interstitial deletion. Am J Med Genet. 2002;113:326-32.

15. McBrien J, Crolla JA, Huang S, Kelleher J, Gleeson J, Lynch SA. Further case of microdeletion of 8q24 with phenotype overlapping Langer-Giedion without TRPS1 deletion. Am J Med Genet A. 2008;146A:1587-92.

16. Pereza N, Severinski S, Ostojic S, Volk M, Maver A, Dekanic $\mathrm{KB}$, et al. Third case of 8q23.3-q24.13 deletion in a patient with Langer-Giedion syndrome phenotype without TRPS 1 gene deletion. Am J Med Genet A. 2012;158A:659-63.

17. Boyle MI, Jespersgaard C, Nazaryan L, Bisgaard AM, Tumer Z. A novel RAD21 variant associated with intrafamilial phenotypic variation in Cornelia de Lange syndrome-review of the literature. Clin Genet 2017;91:647-9. 